\title{
Tradeoff Analysis for Location Update and Paging in Wireless Networks
}

\author{
Yuguang Fang \\ Department of Electrical and Computer Engineering \\ University of Florida, Gainesville, FL 32611-6130
}

\begin{abstract}
Location management plays the central role in providing ubiquitous communications services in the future wireless mobile networks. Location update and paging are commonly used in tracking mobile users on the move, location update is to update a mobile user's current location while the paging is used to locate a mobile user, both will incur signaling traffic in the wireless networks. The more frequent the location updates, the less paging in locating a mobile user, thus there is a tradeoff in terms of signaling cost. Tradeoff analysis of this type has been carried out in the past under exponential assumption for certain time variables. In this paper, we relax all assumptions and analytically derive the average number of location updates during the inter-service time for a movementbased location update scheme under fairly realistic assumption, a number which are required for all tradeoff analysis. We then carry out case studies and numerically demonstrate that the cost function in our analysis indeed has a unique global optimal movement threshold.
\end{abstract}

Keywords-Mobility Management, Location update, Paging, Wireless networks, Mobile networks, Cost analysis.

\section{INTRODUCTION}

In wireless mobile networks, in order to effectively deliver a service to a mobile user, the location of the called mobile user must be determined within a certain time limit (before the service is blocked). There are two basic operations for tracking a mobile user: location update and terminal paging. Location update is the process for the mobile terminals to report their locations to the network, thus all mobiles are actively sending location update messages to keep the network informed. Terminal paging is the process for the network to search the called terminal by sending polling signals to cells close to the last reported location of the called terminal. When an incoming calls to a mobile user (we will use mobile user and mobile terminal interchangeably) arrives, the wireless network simply routes the call to the last reported location of the mobile terminal. Intuitively, the location accuracy depends on the location update frequency, the more often the location updates, the more accurate the location information. If we define the region where a mobile stays as the uncertainty region, then the location update is attempting to minimize the uncertainty region, while the terminal paging is to discover the called terminal in the uncertainty region. The larger the uncertainty region, the higher the paging signaling traffic. Therefore, there is a tradeoff between the location update traffic and the paging traffic. The cost tradeoff analysis has been intensively studied in the current literature ([1], [2], [3], [4], [6], [15], [16], [18]).

Surveying the literature, we have the following couple observations. First, most cost analyses adopted some exponential assumptions for some time variables. For example, in [4], [15], the time between the previously served call arrival and the

This work was supported in part by National Science Foundation Faculty Early Career Award ANI-0093241. time of currently served call ( $t_{c}$ in their notation), also called inter-service time in [10], is assumed to be exponentially distributed. In fact, both [4] and [15] identified the inter-arrival time of call arrivals to a mobile terminal with the inter-service time, thus when the call arrivals to a terminal is assumed to be Poisson, then the inter-service time will be exponentially distributed. However, this identification ignored the busy-line effect: call arrivals to a mobile terminal will be blocked if the mobile terminal is busy serving another call (this will change if voice and data services are integrated, we will not address this situation in this paper), thus the served calls by the mobile is a sampled process from a Poisson process, is most likely not a Poisson process. Moreover, both [4] and [15] ignored the service time for a served call, which is reasonable for PCS networks, because the (voice) calling time is negligible, comparing with the interarrival time between the call arrivals to the mobile terminal. However, in the future wireless mobile networks, a call connection (particularly for data service such as Internet) tends to last longer than voice calls, during the connection period, the mobile does not need to do the location update! Thus, the "inter-service time" is not the right time variable to be used for cost analysis. Rather, it is the mobile terminal idling time, we still use the term inter-service time with the following modifying meaning: it is the time between the end of the previously served call and the arriving instant of the currently served call for a mobile terminal. Obviously, the inter-service time may not be exponentially distributed even if the call arrivals to a mobile terminal is Poisson!

In this paper, we develop techniques to handle the situations when the inter-service time is generally distributed. Our main focus in this paper is to compute the location update cost under generally distributed inter-service time. In the current literature, three location update schemes were proposed and studied ([3], [6]): distance-based location update, movement-based location update and time-based location update. In distancebased locate update scheme, location update will be performed when a mobile terminal moves $d$ cells away from the cell in which the previous location update was performed, where $d$ is a distance threshold. In the movement-based location update scheme, a mobile terminal will carry out a location update whenever the mobile terminal completes $d$ movements between cells, where $d$ is the movement threshold. In the timebased location update scheme, the mobile terminal will update its location every $d$ time units, where $d$ is the time threshold. It has been shown ([6]) that the distance-based location update scheme gives the best result in terms of signaling traffic, however, it may not be practical because a mobile terminal 


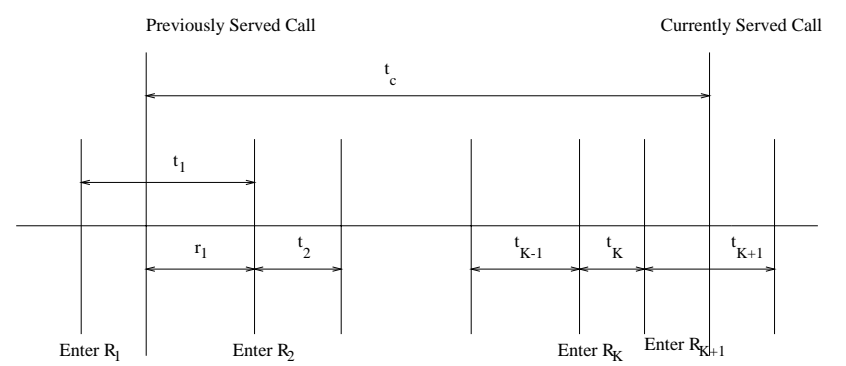

Fig. 1. The time diagram for $K$ cell boundary crossings

has to know its own position information in the network topology. The time-based location update scheme is the simplest to implement, however, unnecessary signaling traffic may result (imagine a stationary terminal for a long period may not need to do any update before it moves). The movement-based location update scheme seems to be the best choice in terms of signaling traffic and implementation. We will concentrate on this scheme for our study in this paper.

This paper is organized as follows. In the next section, we study the probability distribution of number of cell boundary crossing under fairly realistic assumptions. We then give the analytical formula for the average number of location updates during the inter-service time in third section. Tradeoff cost analysis is carried out in the fourth section. We then conclude the paper in the final section.

\section{Probability of the Number of Cell Boundary CROSSINGS}

In order to derive the average number of location updates under the movement-based location update scheme during atypical inter-service time, we first need to know the probability distribution of the number of cell boundary crossings. In this section, we give the analytical results for this distribution.

Assume that the incoming calls to a mobile terminal, say, $\mathcal{T}$, form a Poisson process, the time the mobile terminal stays in a cell (called the cell residence time) has a general non-lattice distribution. We will derive the probability $\alpha(K)$ that a mobile terminal moves across $K$ cells between two served calls arriving to the mobile terminal $\mathcal{T}$. By ignoring the busy line effect and the service time, Lin ([16]) is able to derive an analytical formula for $\alpha(K)$, which has been subsequently used ([4], [16]) for tradeoff analysis for location update and paging. In this section, we assume that the inter-service times are generally distributed and derive an analytic expression for $\alpha(K)$.

Let $t_{1}, t_{2}, \ldots$ denote the cell residence times and $r_{1}$ denotes the residual cell residence time (i.e., the time interval between the time instant the call registers to the network and the time instant the mobile terminal exits the cell). Let $t_{c}$ denote the inter-service time between two consecutive served calls to a mobile terminal $\mathcal{T}$. Figure 1 shows the time diagram for $K$ cell boundary crossings. Suppose that the mobile terminal is in a cell $R_{1}$ when the previous call arrives and accepted by $\mathcal{T}$, it then moves $K$ cells during the inter-service time, and $\mathcal{T}$ resides in the $j$ th cell for a period $t_{j}(1 \leq j \leq K+1)$. We consider a homogeneous wireless mobile network, i.e., all cells in the network are statistically identical. Assume $t_{1}, t_{2}, \ldots$ are independent and identically distributed (iid) with a general probability density function $f(t)$, let $t_{c}$ be generally distributed with probability density function $f_{c}(t)$, and let $f_{r}(t)$ be the probability density function of $r_{1}$. Let $f^{*}(s), f_{c}^{*}(s)$ and $f_{r}^{*}(s)$ denote the Laplace-Stieltjes (L-S) transforms (or simply Laplace transforms) of $f(t), f_{c}(t)$ and $f_{r}(t)$, respectively. Let $E\left[t_{c}\right]=1 / \lambda_{c}$ and $E\left[t_{i}\right]=1 / \lambda_{m}$. From the residual life theorem ([14]), we have

$$
\begin{aligned}
f_{r}(t) & =\lambda_{m} \int_{t}^{\infty} f(\tau) d \tau=\lambda_{m}[1-F(t)], \\
f_{r}^{*}(s) & =\frac{\lambda_{m}}{s}\left[1-f^{*}(s)\right],
\end{aligned}
$$

where $F(t)$ is the distribution function of $f(t)$. It is obvious that the probability $\alpha(K)$ is given by

$$
\begin{aligned}
\alpha(0)= & \left.\operatorname{Pr} t_{c} \leq r_{1}\right], \quad K=0, \\
\alpha(K)= & \operatorname{Pr}\left[r_{1}+t_{2}+\cdots+t_{K}<t_{c}\right. \\
& \left.\leq r_{1}+t_{2}+\cdots+t_{K+1}\right], \quad K \geq 1
\end{aligned}
$$

We first calculate $\alpha(0)$. Since the Laplace transform of $\int_{t}^{\infty} f_{r}(\tau) d \tau$ is $\left(1-f_{r}^{*}(s)\right) / s$, from (2), the inverse Laplace transform and the independence of $r_{1}$ and $t_{c}$, we obtain

$$
\begin{aligned}
\alpha(0) & =\int_{0}^{\infty} \operatorname{Pr}\left(r_{1} \geq t\right) f_{c}(t) d t \\
& =\frac{1}{2 \pi j} \int_{\sigma-j \infty}^{\sigma+j \infty} \frac{1-f_{r}^{*}(s)}{s} f_{c}^{*}(-s) d s
\end{aligned}
$$

where $\sigma$ is a sufficiently small positive number which is appropriately chosen for inverse Laplace transform.

For $K>0, \alpha(K)$ is computed as follows. First, we need to compute $\operatorname{Pr}\left(r_{1}+t_{2}+\cdots+t_{k} \leq t_{c}\right)$ for any $k>0$. Let $\xi=r_{1}+t_{2}+\cdots+t_{k}$. We can obtain

$$
\begin{aligned}
& \operatorname{Pr}\left(r_{1}+t_{2}+\cdots+t_{k} \leq t_{c}\right) \\
= & \frac{1}{2 \pi j} \int_{\sigma-j \infty}^{\sigma+j \infty} \frac{f_{r}^{*}(s)\left[f^{*}(s)\right]^{k-1}}{s} f_{c}^{*}(-s) d s .
\end{aligned}
$$

Taking this into (3), we obtain

$\alpha(K)=\frac{1}{2 \pi j} \int_{\sigma-j \infty}^{\sigma+j \infty} \frac{f_{r}^{*}(s)\left[f^{*}(s)\right]^{K-1}\left[1-f^{*}(s)\right]}{s} f_{c}^{*}(-s) d s$

Let $\sigma_{c}$ denote the set of poles of $f_{c}^{*}(-s)$ in the right half complex plane, then applying the Residue Theorem ([14]), we obtain

Theorem 1. If the probability density function of inter-service time has only finite possible isolated poles (which is the case when it has a rational Laplace transform), then the probability 
$\alpha(K)$ that a mobile terminal moves across $K$ cells during the inter-service time is given by

$$
\begin{aligned}
\alpha(0) & =\frac{1}{2 \pi j} \int_{\sigma-j \infty}^{\sigma+j \infty} \frac{1-f_{r}^{*}(s)}{s} f_{c}^{*}(-s) d s \\
& =-\sum_{p \in \sigma_{c}} \operatorname{Res}_{s=p} \frac{\left.1-f_{r}^{*}(s)\right)}{s} f_{c}^{*}(-s), \\
\alpha(K) & =\frac{1}{2 \pi j} \int_{\sigma-j \infty}^{\sigma+j \infty} \frac{f_{r}^{*}(s)\left[1-f^{*}(s)\right]\left[f^{*}(s)\right]^{K-1}}{s} f_{c}^{*}(-s) d s \\
& =-\sum_{p \in \sigma_{c}} \operatorname{Res}_{s=p} \frac{f_{r}^{*}(s)\left[1-f^{*}(s)\right]\left[f^{*}(s)\right]^{K-1}}{s} f_{c}^{*}(-s)
\end{aligned}
$$

where Res denotes the residue at poles $s=p$ and $f_{r}^{*}(s)=$ $\lambda_{m}\left(1-f^{*}(s)\right) / s$.

\section{AVERAGE Number of LOCATION Updates}

Consider the movement-based location update scheme. Let $d$ denote the movement threshold, i.e., a mobile terminal will perform a location update whenever the mobile terminal makes $d$ movements (equal to the number of serving cell switching) after the last location update. Then, the average number of location updates during an inter-service time interval under movementbased location update scheme can be expressed as ([4])

$$
N_{u}(d)=\sum_{i=1}^{\infty} i \sum_{k=i d}^{(i+1) d-1} \alpha(k)
$$

In what follows in this section, we present the computation for $N_{u}(d)$. Let

$$
S(n)=\sum_{k=1}^{n-1} \alpha(k)
$$

then, from Theorem 1, we obtain

$$
S(n)=\frac{1}{2 \pi j} \int_{\sigma-j \infty}^{\sigma+j \infty} \frac{f_{r}^{*}(s)\left[1-\left(f^{*}(s)\right)^{n-1}\right]}{s} f_{c}^{*}(-s) d s
$$

Moreover, we have

$$
\begin{aligned}
& \sum_{k=1}^{N} s(k d) \\
& =\sum_{k=1}^{N} \frac{1}{2 \pi j} \int_{\sigma-j \infty}^{\sigma+j \infty} \frac{f_{r}^{*}(s)\left[1-\left(f^{*}(s)\right)^{k d-1}\right]}{s} f_{c}^{*}(-s) d s \\
& =\frac{1}{2 \pi j} \int_{\sigma-j \infty}^{\sigma+j \infty} \frac{f_{r}^{*}(s)}{s} \\
& \times\left\{N-\frac{\left(f^{*}(s)\right)^{d-1}\left[1-\left(f^{*}(s)\right)^{N d}\right]}{1-\left(f^{*}(s)\right)^{d}}\right\} f_{c}^{*}(-s) d s
\end{aligned}
$$

Thus, from (6), (7) and (8), after complicated mathematical manipulations, we obtain

$$
N_{u}(d)=\sum_{i=1}^{\infty} i[S((i+1) d)-S(i d)]
$$

$$
\begin{aligned}
= & \lim _{N \rightarrow \infty}\left\{\sum_{i=1}^{N} i[S((i+1) d)-S(i d)]\right\} \\
= & \lim _{N \rightarrow \infty}\left\{N S((N+1) d)-\sum_{i=1}^{N} S(i d)\right\} \\
= & \frac{1}{2 \pi j} \int_{\sigma-j \infty}^{\sigma+j \infty} \frac{f_{r}^{*}(s)\left(f^{*}(s)\right)^{d-1}}{s\left[1-\left(f^{*}(s)\right)^{d}\right]} f_{c}^{*}(-s) \\
- & \lim _{N \rightarrow \infty} \frac{1}{2 \pi j}\left\{\int_{\sigma-j \infty}^{\sigma+j \infty} \frac{f_{r}^{*}(s)\left[N+1-N\left(f^{*}(s)\right)^{d}\right]}{s\left[1-\left(f^{*}(s)\right)^{d}\right]}\right. \\
& \left.\times\left[f^{*}(s)\right]^{(N+1) d-1} f_{c}^{*}(-s) d s\right\} \\
= & \frac{1}{2 \pi j} \int_{\sigma-j \infty}^{\sigma+j \infty} \frac{f_{r}^{*}(s)\left(f^{*}(s)\right)^{d-1}}{s\left[1-\left(f^{*}(s)\right)^{d}\right]} f_{c}^{*}(-s) d s
\end{aligned}
$$

In summary, we obtain

Theorem 2: The average number of location updates $N_{u}(d)$ is given by

$$
N_{u}(d)=\frac{1}{2 \pi j} \int_{\sigma-j \infty}^{\sigma+j \infty} \frac{f_{r}^{*}(s)\left(f^{*}(s)\right)^{d-1}}{s\left[1-\left(f^{*}(s)\right)^{d}\right]} f_{c}^{*}(-s) d s .
$$

If $f_{c}^{*}(s)$ has a finite number of poles, then we have

$$
N_{u}(d)=-\sum_{p \in \sigma_{c}} \operatorname{Res}_{s=p} \frac{f_{r}^{*}(s)\left(f^{*}(s)\right)^{d-1}}{s\left[1-\left(f^{*}(s)\right)^{d}\right]} f_{c}^{*}(-s),
$$

where $\sigma_{c}$ is the set of poles of $f_{c}^{*}(-s)$.

\section{TRadeoff Cost Analysis}

In this section, we present some results for the tradeoff analysis under movement-based location update scheme and some paging scheme with the linear cost functional for illustration purpose.

\section{A. Location Update Cost}

If $U$ denotes the unit cost for location update, then total location update cost is given by

$$
\mathcal{C}_{u}(d)=-U \sum_{p \in \sigma_{c}} \operatorname{Res}_{s=p} \frac{f_{r}^{*}(s)\left(f^{*}(s)\right)^{d-1}}{s\left[1-\left(f^{*}(s)\right)^{d}\right]} f_{c}^{*}(-s),
$$

If the inter-service time $t_{c}$ is exponentially distributed with parameter $\lambda_{c}$, then $f_{c}^{*}(s)=\lambda_{c} /\left(s+\lambda_{c}\right)$, from (11), we can easily obtain

$$
\mathcal{C}_{u}(d)=\frac{U f_{r}^{*}\left(\lambda_{c}\right)\left[f^{*}\left(\lambda_{c}\right)\right]^{d-1}}{1-\left[f^{*}\left(\lambda_{c}\right)\right]^{d}}
$$

This result was obtained in [15] (noticing that $f_{r}^{*}(s)=\lambda_{m}(1-$ $\left.\left.f^{*}(s)\right) / s\right)$.

If the inter-service time $t_{c}$ is Erlang distributed with parameter $\left(p, \lambda_{c}\right)$, i.e.,

$$
f_{c}(t)=\frac{\left(p \lambda_{c}\right)^{p} t^{p-1}}{(p-1) !} e^{-p \lambda_{c} t}, f_{c}^{*}(s)=\left(\frac{p \lambda_{c}}{s+p \lambda_{c}}\right)^{p},
$$


then the location update cost is given by

$$
\mathcal{C}_{u}(d)=\frac{U(-1)^{p-1}\left(p \lambda_{c}\right)^{p}}{(p-1) !} g^{(p-1)}\left(p \lambda_{c}\right)
$$

where $g^{(i)}(x)$ denotes the $i$ th derivative of function $g(x)$ at point $x$ and

$$
g(s)=\frac{f_{r}^{*}(s)\left(f^{*}(s)\right)^{d-1}}{s\left[1-\left(f^{*}(s)\right)^{d}\right]} .
$$

\section{B. Paging Cost}

We consider the paging strategy used in [15] for our case study. Consider the hexagonal layout for the wireless network, all cells are statistically identical. According to the movementbased location update scheme, a mobile terminal moves at most $d$ cells away from the previous position where it performs the last location update. Thus, a mobile terminal will surely be located in a cell which is less than $d$ cell away from the previously reported position. If we page in the circular area with $d$ cells as radius and with the previously reported position as the center, then we can definitely find the mobile terminal. Thus, this paging scheme is the most conservative among all paging. If we let $P$ denote the unit cost for each paging in a cell, the maximum paging cost for this paging scheme is given by ([15])

$$
\mathcal{C}_{p}(d)=P(1+3 d(d-1))
$$

\section{Total Cost}

The unit costs (cost factors) $U$ and $P$ can be chosen to reflect the significance of the signaling (they may be significantly different from each other because they use different network resources). Given $U, P$ and the movement threshold $d$, the total cost for location update and paging will be given by

$$
\mathcal{C}(d)=\mathcal{C}_{u}(d)+\mathcal{C}_{p}(d)
$$

\section{Numerical Analysis}

In this subsection, we present some illustrative examples to show the behavior of overall cost function with respect to various parameters. We assume that the cell residence time is Gamma distributed with the following probability density function and the Laplace transform:

$$
f(t)=\frac{\left(\gamma \lambda_{m}\right)^{\gamma} t^{\gamma-1}}{\Gamma(\gamma)} e^{-\gamma \lambda_{m} t}, f^{*}(s)=\left(\frac{\gamma \lambda_{m}}{s+\gamma \lambda_{m}}\right)^{\gamma} .
$$

The mean is $1 / \lambda_{m}$ and the variance is $1 /\left(\gamma \lambda_{m}^{2}\right)$. We assume that the inter-service time is Erlang distributed with parameters $\left(p, \lambda_{c}\right)$, its mean is $1 / \lambda_{c}$ and its variance $1 /\left(p \lambda_{c}^{2}\right)$. We first carry out the similar studies as in [15] and observe the property of the total cost function with respect to the parameters such as the call-to-mobility ratio (CMR) $\theta=\lambda_{c} / \lambda_{m}$ and the variance of the cell residence time to compare our results with those given in [15]. We then present some more study for the case when the inter-service time is not exponentially distributed.

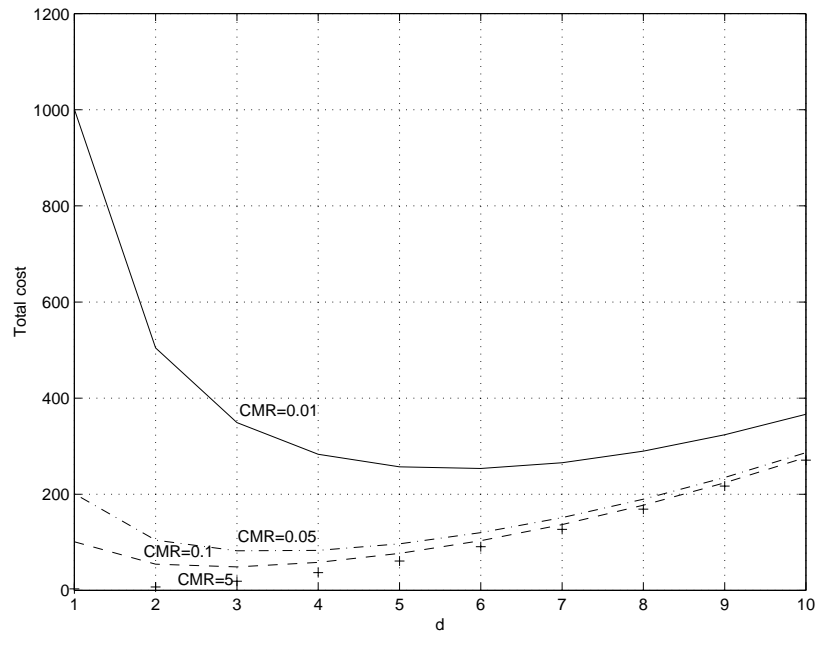

Fig. 2. Total cost vs. the movement thresholds: varying call-to-mobility ratio (CMR)

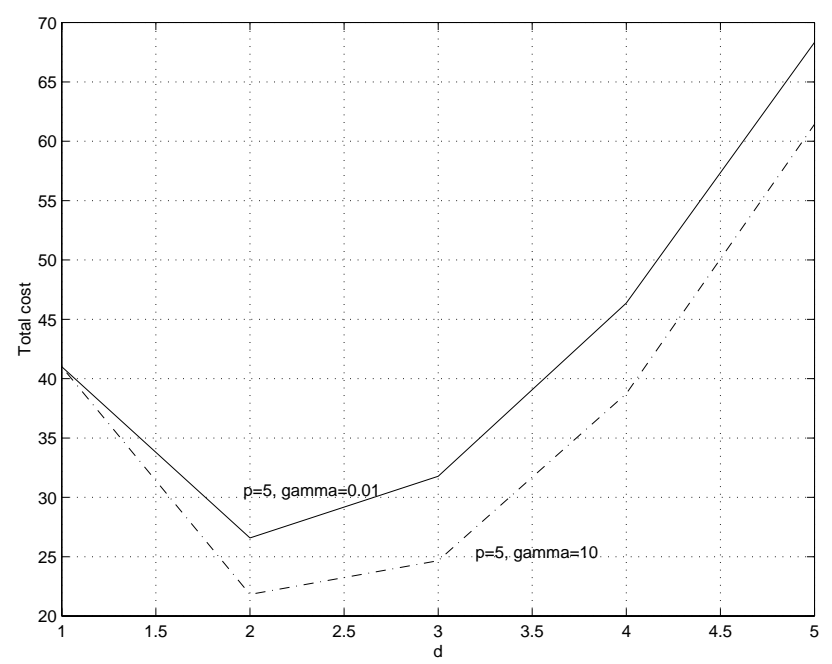

Fig. 3. Total cost vs. the movement threshold for Erlang inter-service time: varying variance of cell residence time

Figure 2 shows the cost function vs. movement threshold for various call-to-mobility ratios. As we observe from the figure, the cost function is also convex, the global minimum (the optimal movement threshold) $d$ decreases as the CMR increases. This is also intuitively understandable because higher CMR indicates more location updates are necessary.

Figure 3 shows that the cost function vs. the movement threshold for varying variance of the cell residence time (or $\gamma$ ), where we choose $P=1$ and $U=20$ and the inter-service time is Erlang distributed with $p=5$. Figure 3 shows significant difference for different values of $\gamma$ (hence the variances of the cell residence time), the total cost is in fact sensitive to $\gamma$.

In Figure 4, we fix the average cell residence time and the average inter-service time, and change the variances of cell residence time and inter-service time (i.e., $\gamma$ and $p$ ), we again observe the significant differences between the total costs (even 


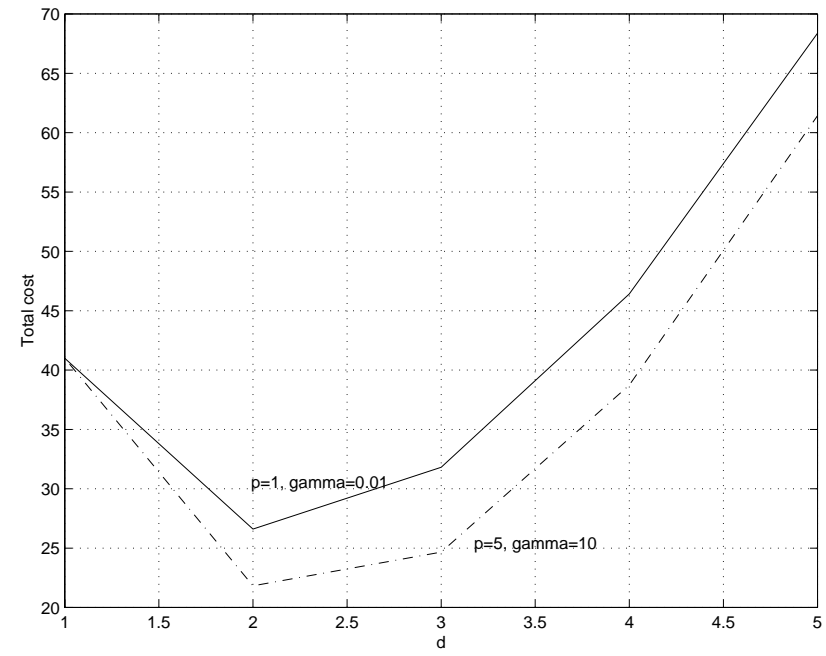

Fig. 4. Total cost vs. the movement threshold for exponential inter-service time: varying variances of both cell residence time and inter-service time

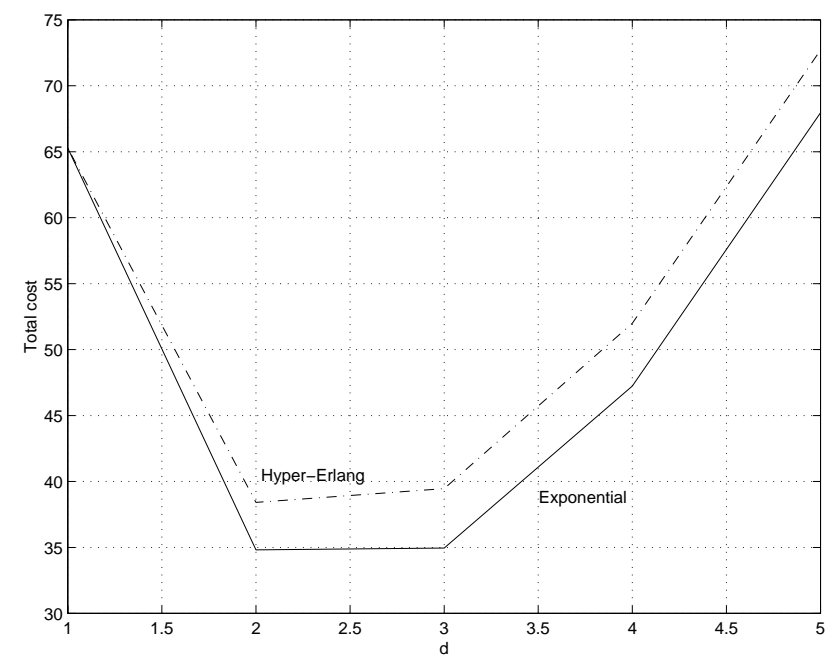

Fig. 5. Total cost vs. the movement threshold when the inter-service time is exponentially distributed and hyper-Erlang distributed, respectively

the optimal costs, i.e., the one corresponding to the optimal thresholds).

Next, we study the sensitivity issue of the total cost with respect to the variance of inter-service time. Figure 5 shows the plots when the inter-service time $t_{c}$ is exponentially distributed and hyper-Erlang distributed with the same mean, respectively ([8]). In this example, we assume that hyper-Erlang distribution has the following parameters: $\alpha_{1}=0.15, \alpha_{2}=0.85, m_{1}=1$, $m_{2}=20, \eta_{1}=0.07, \eta_{2}=5000$, thus the hyper-Erlang distribution has mean $1 / \lambda_{c}=\alpha_{1} / \eta_{1}+\alpha_{2} / \eta_{2}=2.143$ and with variance $\alpha_{1} /\left(m_{1} \eta_{1}^{2}\right)+\alpha_{2} /\left(m_{2} \eta_{2}^{2}\right)=30.6122$, while the exponential distribution will have mean $1 / \lambda_{c}=2.143$ and variance $1 / \lambda_{c}^{2}=4.5926$. From the figure, we observe that total cost is indeed sensitive to the variance of the inter-service time (the total cost for hyper-Erlang case can be $12 \%$ higher.

\section{CONCLUSION}

In this paper, we have performed the tradeoff analysis for the movement-based location update and paging for the wireless mobile networks. We obtain analytically the crucial quantity, the average number of location updates during an inter-service time, used in all cost analysis, under fairly general assumptions. The analytical results and analytical approach for the cost analysis developed in this paper can be very useful in the design of mobility management for the future wireless mobile networks. Our study shows that the total cost of the location update and paging is a convex function of movement threshold, hence we can always find the unique optimal threshold.

\section{ACKNOWLEDGMENT}

The author would express his sincere appreciation to FarEastone in Taiwan for their help during the writing of this paper.

\section{REFERENCES}

[1] A. Abutaleb and V. O. K. Li, "Paging strategy optimization in personal communication systems," Wireless Networks, vol.3, pp.195-204, 1997.

[2] A. Abutaleb and V. O. K. Li, "Location update optimization in personal communication systems," Wireless Networks, vol.3, pp.205-216, 1997.

[3] I.F. Akyildiz and J.S.M. Ho, "Dynamic mobile user location update for wireless PCS networks," Wireless Networks, vol.1, pp.187-196, 1995.

[4] I.F. Akyildiz, J.S.M. Ho and Y.-B. Lin, "Movement-based location update and selective paging for PCS networks," IEEE/ACM Trans. Networking, vol.4, no.4, pp.629-638, 1996.

[5] I.F. Akyildiz, J. McNair, J.S.M. Ho, H. Uzunalioglu and W. Wang, "Mobility management in next-generation wireless systems," Proc. of the IEEE, vol.87, no.8, pp.1347-1384, August 1999.

[6] A. Bar-Noy, I. Kessler and M. Sidi, "Mobile users: to update or not update?" Wireless Networks, vol.1, no.2, pp.175-186, July 1994.

[7] D. C. Cox, "Wireless personal communications: what is it?" IEEE Personal Comm. Mag., pp.20-35, April 1995.

[8] Y. Fang and I. Chlamtac, "Teletraffic analysis and mobility modeling for PCS networks," IEEE Transactions on Communications, Vol. 47, No. 7, pp. 1062-1072, July 1999.

[9] Y. Fang, I. Chlamtac and H. Fei, "Analytical results for optimal choice of location update interval for mobility database failure restoration in PCS networks," IEEE Transactions on Parallel and Distributed Systems, vol.11, no.6, pp.615-624, June 2000.

[10] Y. Fang, I. Chlamtac and Y. B. Lin, "Portable movement modeling for PCS networks" To appear in IEEE Trans. Veh. Tech.

[11] Y. Fang, I. Chlamtac and Y. B. Lin, "Call performance for a PCS network," IEEE JSAC, vol.15, no.8, pp.1568-1581, 1997.

[12] D. Hong and S. S. Rappaport, "Traffic model and performance analysis for cellular mobile radio telephone systems with prioritized and nonprioritized handoff procedures," IEEE Trans. Veh. Tech., vol.35, no.3, pp.7792, 1986.

[13] B. Jabbari, "Teletraffic aspects of evolving and next-generation wireless communication networks," IEEE Comm. Mag., pp.4-9, December 1994.

[14] L. Kleinrock, Queueing Systems: Theory, Volume I, John Wiley \& Sons, New York, 1975.

[15] J. Li, H. Kameda and K. Li, "Optimal dynamic mobility management for PCS networks," IEEE/ACM Transactions on Networking, vol.8, no.3, pp.319-327, 2000

[16] Y. B. Lin, "Reducing location update cost in a PCS network," IEEE/ACM Transactions on Networking, vol.5, no.1, pp.25-33, 1997.

[17] Y. B. Lin, S. Mohan and A. Noerpel "Queueing priority channel assignment strategies for handoff and initial access for a PCS network," IEEE Trans. Veh. Technol., vol.43, no.3, pp.704-712, 1994.

[18] C. Rose and R. Yates, "Minimizing the average cost of paging under delay constraints," Wireless Networks, vol.1, no.2, pp.211-219, 1995. 